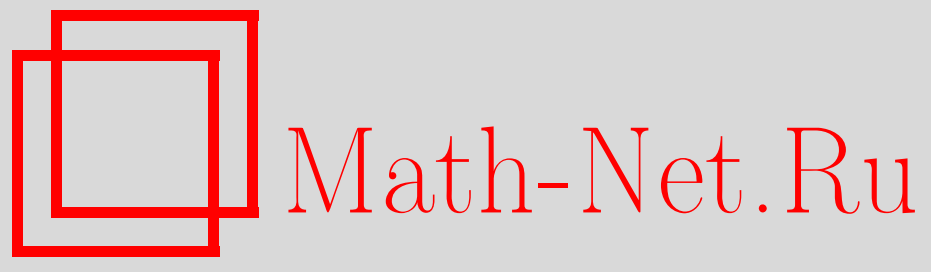

В. П. Маслов, Нестандартный анализ, парастатистики и фракталы, ТМФ, 2007, том 153, номер 2, 262-270

DOI: https://doi.org/10.4213/tmf6137

Использование Общероссийского математического портала Math-Net.Ru подразумевает, что вы прочитали и согласны с пользовательским соглашением http://www . mathnet.ru/rus/agreement

Параметры загрузки:

IP: 54.198 .187 .58

26 апреля 2023 г., 18:16:46

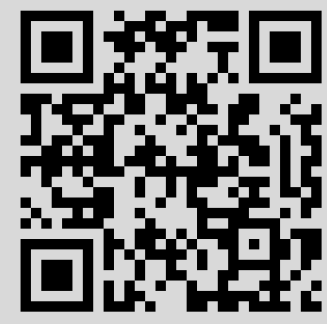




\section{НЕСТАНДАРТНЫЙ АНАЛИЗ, ПАРАСТАТИСТИКИ И ФРАКТАЛЫ}

Для оценки расходящихся интегралов удобно применять, с одной стороны, идеи нестандартного анализа, а с другой стороны, аппроксимацию интеграла специальной решетчатой моделью, которую можно трактовать как квантование пространства. Эти методы применяются в случае нецелых (фрактальных) и отрицательных (дырочных) размерностей. Приводятся уточняющие формулы, в частности, для спектра фликкер-шумов.

Ключевые слова: нестандартный анализ, парастатистики, фликкер-шум, квантование пространства.

При определении амплитуд в фейнмановском континуальном интеграле рассматриваются случайные амплитуды, "распределенные одинаково равномерно"1). Как известно, мера Лебега не продолжается на множество непрерывных траекторий, поэтому естественно называть такой несуществующий набор случайных функций "амплитудами Фейнмана, распределенными одинаково равномерно", и определять их в рамках нестандартного анализа и перехода к решетчатой модели.

Рассмотрим плотность $t^{-s}$ и расходящиеся интегралы (переход к сферическим координатам в $D$-мерном интеграле показывает, что размерность $D$ пространства равна $s+1)$

$$
\int_{0}^{\Omega} \frac{x(t)}{t^{s}} d t=\omega,
$$

где $\omega$ и $\Omega$ - бесконечно большие (нестандартные) числа, и

$$
\int_{0}^{\Omega} \frac{x(t) \lambda(t)}{t^{s}} d t \leqslant c(\omega),
$$

где $x(t) \geqslant 0 ; \lambda(t)$ - заданная монотонно возрастающая функция, $\lambda^{\prime}(t) \geqslant \sigma>0, c(\omega)-$ бесконечно большая функция от $\omega$ (инфракрасная катастрофа при $s \geqslant 1$ и ультрафиолетовая при $s \leqslant-1)$. Предположим, что "амплитуды Фейнмана $x(t) \lambda(t) / t^{s}$ равномерно распределены" в области, ограниченной условиями (1) и (2), т.е. "амплитуды

1) Здесь и ниже мы ставим кавычки, подразумевая, что говорим на физическом "жаргоне".

* Московский государственный университет, Москва, Россия. E-mail: v.p.maslov@mail.ru 
$x(t) \lambda(t) / t^{s}$ равновероятны для всех случайных $x(t)$, удовлетворяющих условиям (1) и (2)" (см. [1]).

Произведем формальную замену масштаба, "растянув" интервал интегрирования $(0,1)$ на отрезок $(0, n)$, где $n \gg 1$ :

$$
n^{s-1} \int_{0}^{n \Omega} \frac{\tilde{x}(\tau) \tilde{\lambda}(\tau)}{\tau^{s}} d \tau, \quad \tau=t n, \quad \tilde{x}\left(\frac{\tau}{n}\right)=x(t), \quad \tilde{\lambda}\left(\frac{\tau}{n}\right)=\lambda(t) .
$$

Согласно формуле Эйлера

$$
n^{s-1} \int_{1}^{n \Omega} \frac{\tilde{x}(\tau) \widetilde{\lambda}(\tau)}{\tau^{s}} d s \sim \sum_{i=s}^{n[\Omega]} \widetilde{\lambda}(i) \tilde{x}(i) \rho_{i} \quad\left(\bmod \frac{1}{n}\right)
$$

где $n$ - бесконечно большое число, $\rho_{i} \sim(n / i)^{s},[\Omega]$ - целая часть $\Omega$.

Таким образом, мы аппроксимируем интегралы (1) и (2) сеткой (решеткой) с шагом 1 на интервале $(1, n)$, где $n$ - бесконечно большое число. Все плотности $1 / t^{s}$ квантуются последовательностью (по $\left.\bmod \frac{1}{n}\right)$

$$
\rho_{i} \cong\left(\frac{n}{i}\right)^{s}, \quad i=1, \ldots, n \text {. }
$$

Теперь понятие "одинаково равномерного распределения" (мера Лебега) определяется точно как равновозможность всех вариантов, удовлетворяющих условиям (1) и $(2)$.

В квантовой теории поля, как известно, ряд трудностей может быть преодолен, если перейти к решетчатым моделям, т.е. проквантовать пространство. Недавно Манин [2] поднял вопрос об отрицательной плотности множества. Он, по-видимому, первый обратил внимание на то, что использование бра- и кет-векторов Дирака для определения обобщенной функции может быть интерпретировано как введение в теорию плотности, отвечающей отрицательной размерности.

Умножение на координату двойственно в смысле преобразования Фурье оператору дифференцирования. Поэтому мы можем говорить о двойственных $n$ раз дифференцируемых функциях соболевского пространства $W_{2}^{n}$. "Сопряженным" к такому пространству является пространство $W_{2}^{-n}$ обобщенных функций по Соболеву. Точно так же мы можем определить через "скалярное произведение" функции из пространства $W_{2}^{s}$, где $s$ - нецелое положительное число, и $W_{2}^{-s}$ как "скалярно" сопряженное к нему. Аналогично можно поступить с плотностью (весом) пары $r^{s}$ и $r^{-s}$, используя, например, для представления функций из пространства $W_{2}^{s}$ ядро Рисса или потенциал Бесселя.

Если говорить о непрерывном случае, который рассматривал Манин, то можно огрубленно сказать, что если есть “ультрафиолетовая расходимость", то размерность положительная, а если есть "инфракрасная расходимость", то размерность отрицательная. В квантовой теории поля также используется аналитическое продолжение 
размерности для регуляризации ряда фейнмановских диаграмм (см. [3] и приведенную там литературу).

Чтобы перейти к дискретной размерности, рассмотрим одномерное уравнение Шредингера

$$
-\frac{h^{2}}{2 m} \frac{d^{2} \Psi_{k}}{d x^{2}}+u(x) \Psi_{k}=E_{k} \Psi_{k}
$$

с дискретным спектром $\left\{E_{n}\right\}, u(x) \in C^{\infty}, u(x) \rightarrow \infty$ при $x \rightarrow \infty$ быстрее, чем $x^{\alpha}$ при $\alpha \geqslant \delta>0$. Ему отвечает одномерный оператор Гамильтона

$$
\widehat{H}_{x}=-\frac{h^{2}}{2 m} \frac{d^{2}}{d x^{2}}+u(x) .
$$

В $D$-мерном случае

$$
\sum_{i=1}^{D} \frac{h^{2}}{2 m} \frac{\partial^{2} \Psi_{k}}{\partial x_{i}^{2}}+u\left(x_{i}\right) \Psi_{k}=\widetilde{E}_{k} \Psi_{k} .
$$

Здесь собственное значение $\widetilde{E}_{k}$ является $D$-мерной суммой значений $E_{k}$ :

$$
\widetilde{E}_{k}=\sum_{i=1}^{D} E_{n_{i}} .
$$

Как известно, на элемент фазового объема

$$
\frac{\Delta x_{1} \Delta p_{1} \Delta x_{2} \Delta p_{2} \ldots \Delta x_{D} \Delta p_{D}}{(2 \pi h)^{D}}
$$

приходится $\Delta N^{D}$ квантовых состояний [4]. Отсюда число собственных значений $\widetilde{E}_{k} \leqslant \mathcal{E}$, где $\mathcal{E}$ - заданное, не зависящее от $h$ число, в главном члене имеет порядок $h^{-D}$ при $h \rightarrow 0$. Тогда если $h=1$, а $E_{s} \leqslant \mathcal{E} \rightarrow \infty$, то число собственных значений $\widetilde{E}_{k}^{(D)} \sim \mathcal{E}^{D-1}$. В функциональном анализе это хорошо известный факт.

Более общее утверждение (лемма 1) будет высказано как гипотеза.

Пусть задана последовательность положительных чисел $\left\{\lambda_{i}\right\}$

$$
\left\{\lambda_{i}\right\}=\lambda_{1}, \lambda_{2}, \ldots, \lambda_{n}, \quad \lambda_{1}<\lambda_{2}<\cdots<\lambda_{n} ; \quad \lambda_{n} \leqslant \mathcal{E}(n) ; \quad n \rightarrow \infty, \quad \mathcal{E}(n) \rightarrow \infty .
$$

Разность $\lambda_{i+1}-\lambda_{i}=O(1)$, т.е. $\lambda_{i+1}-\lambda_{i} \geqslant \delta>0$ при $n \rightarrow \infty$, где $\delta$ не зависит от $n$ для всех $i$.

Рассмотрим пространство размерности $k$ и сопоставим ему $k$ последовательностей $\left\{\lambda_{i}\right\}$ и все комбинации сумм длины $\sum_{j=1}^{k} \lambda_{i_{j}} \leqslant \mathcal{E}(n)$.

ЛЕмма 1. Плотность значения этих комбинаций, т.е. число этих значений, имеет порядок $O\left(n^{D-1}\right)$.

Нас будут в основном интересовать два случая: $\lambda_{i}=i$ и $\lambda_{i}=i^{2}$. В первом случае сумма $\sum_{j=1}^{D} \lambda_{i_{j}}$ вычисляется точно для $u(x)=\alpha x^{2}, \alpha=$ const, и является целым числом с кратностью, равной

$$
q_{i}(D)=\frac{i(i+1) \ldots(i+D-2)}{(D-1) !} .
$$


Целочисленность согласуется с формулой (4).

Во втором случае проблема сводится к числу целых точек в $D$-мерном шаре. Кроме того, эта задача эквивалентна случаю $u(x)=0$ в уравнении (5) с нулевыми краевыми условиями и подпадает под общий случай. В этом случае известен лишь порядок $O\left(n^{D-1}\right)$, приведенный в лемме 1 . Но для приложений более точного результата не требуется (см. ниже).

Аналитическое продолжение $q_{i}(D)$ на нецелые и отрицательные $k$ имеет вид

$$
q_{i}(D)=\frac{\Gamma(i+D-1)}{\Gamma(D) \Gamma(i)} .
$$

Если $D \leqslant 0$, то мы называем число $|D|$ дырочной размерностью.

"Квантование" пространств с отрицательной размерностью проводится точно так же [5], как и с положительной, по той же формуле (1), поскольку Г-функция аналитически продолжается для отрицательных $D$. Если $D$ - целое отрицательное число, то при $i=1,2, \ldots,|D+1|$ имеют место простые полюсы, которые являются дискретным аналогом инфракрасной катастрофы. Можно также считать эти точки конденсатом. Поэтому имеют смысл только значения плотности при $i>|D|$, и после нормировки

$$
\rho_{i}(D)=\frac{q_{i}(D)}{\max _{i} q_{i}(D)}, \quad 1 \leqslant i \leqslant n,
$$

они равны

$$
\rho_{i}=\frac{1}{i(i-1)(i-2) \ldots(i-D-1)} .
$$

Если $D$ - отрицательное нецелое число, то при $i>|D|$

$$
\rho_{i}=\frac{\Gamma(i+D-1)}{(i-1) !},
$$

а при $i<D$ значения $\rho_{i}$ меняют знаки. В силу формулы Эйлера выполняется соотношение (4).

Имеет место следующая теорема о распределении парастатистик ${ }^{2)}$ [6].

Teорема. Пусть размерность равна $D$ и пусть задан уровень энергии $\mathcal{E}$. Рассмотрим множество всех равновозможных вариантов распределения частии $N_{i}$ на уровнях энергии $\lambda_{i}=i$ таких, что: 1) $\left.\left.N_{i} \leqslant R ; 2\right) \sum_{i} N_{i} \lambda_{i} \leqslant \mathcal{E} ; 3\right) \sum_{i} N_{i}=N$.

Обозначим через $\beta$ и $\sigma$ решения системы

$$
\begin{gathered}
\sum_{i=\max \{1,1-[D]\}}^{n} \lambda_{i}\left\{\frac{\rho_{i}(D)}{e^{\beta \lambda_{i}+\sigma}-1}-\frac{(R+1) \rho_{i}(D)}{e^{\left(\beta \lambda_{i}+\sigma\right)(R+1)}-1}\right\}=E, \\
\sum_{i=\max \{1,1-[D]\}}^{n}\left\{\frac{\rho_{i}(D)}{e^{\beta \lambda_{i}+\sigma}-1}-\frac{(R+1) \rho_{i}(D)}{e^{\left(\beta \lambda_{i}+\sigma\right)(R+1)}-1}\right\}=\alpha .
\end{gathered}
$$

2) Мы специально оставляем в формулировках $\lambda_{i}$, чтобы была видна связь с парастатистиками и чтобы была ясна идейная связь с ниже приводимым случаем $\lambda_{i}=i^{2}$. 
Тогда для произвольных $C_{0}>0, \gamma \in[0,1), \varepsilon \in(0,(1-\gamma) / 2) u \varepsilon_{1}<2 \varepsilon$ существуют такие числа $C, K>0$, не зависящие от $s$, что для любых заданных попарно непересекающихся подмножеств $J_{1}, \ldots, J_{k} \subset\{1, \ldots, s\}$, где $k \leqslant C_{0} s^{\gamma}$, выполнена оценка вероятности

$$
\begin{aligned}
\mathrm{P}\left(\sum_{m=1}^{k} \mid\right. & \left.\sum_{j \in J_{m}} N_{j}-\sum_{j \in J_{m}}\left\{\frac{\rho_{j}(D)}{e^{\beta \lambda_{j}+\sigma}-1}-\frac{(R+1) \rho_{j}(D)}{e^{\left(\beta \lambda_{j}+\sigma\right)(R+1)}-1}\right\} \mid \geqslant \frac{N}{n^{(1-\gamma) / 2-\varepsilon}}\right)< \\
& <C e^{-K n^{\gamma+\varepsilon_{1}}} .
\end{aligned}
$$

Ниже мы будем рассматривать случай $R=\infty$, т.е. бозе-статистику. Таким образом, большинство равновозможных вариантов скапливается около коммулятивного бозе-распределения, т.е. число всех частиц, приходящихся на уровни $\lambda_{i} \leqslant \lambda_{k}$ при заданном $k$, отвечает формуле для бозе-распределения с точностью, указанной в теореме. Иными словами, большинство вариантов, отвечающих условиям (1) и (2), слабо сходятся к бозе-распределению, т.е. колеблются около бозе-распределения.

Приведем пример из лингвостатистики. Рассмотрим частотный словарь, отвечающий массиву текстов с числом словоупотреблений $M$. Пусть $N_{i}$ - количество слов, число встречаемости которых в данном текстовом массиве равно $\omega_{i}$. Тогда $\sum_{i} N_{i}=N$, где $N$ - число различных слов в словаре, составленном по массиву текстов $M$, а

$$
\sum_{i=1}^{N} \omega_{i} N_{i}=M
$$

Число слов $N_{1}$, встречающихся в массиве текстов только один раз, как правило, имеет порядок $O(N)$, т.е. $N_{1} \rightarrow \infty$ при $N \rightarrow \infty$. Поэтому можно полагать, что размерность $D=0$.

Переходя от сумм к интегралу и имея в виду, что $\beta \ll 1$, а $\sigma=\beta \nu$, где $|\nu| \sim 1-$ химический потенциал, и для не очень больших $\omega$ можно брать первый член разложения по $\beta \rightarrow 0$, выбрав в качестве одного из множеств $J_{m}$ интервал $\left(i_{0}, i_{1}\right)$ в средней области частотного словаря, получим

$$
\sum_{i=i_{0}}^{i_{1}} N_{i} \sim \int_{\omega_{0}}^{\omega} \frac{d \omega}{\omega(\beta \omega+\beta \nu)}=a \ln \frac{c \omega}{1+b \omega}
$$

где $a, b, c$ - константы, выражаемые через $\beta, \nu, \omega_{0}$ (см. [7]).

Именно средняя область частотного словаря характерна для того или иного писателя. Так, для разных кусков описания природы в романах "Тихий Дон" и "Поднятая целина" температура и химический потенциал меняются не сильно, в то время как для произведений $\Phi$. Крюкова, которому некоторые приписывают авторство "Тихого Дона", химический потенциал отличается от первых в 100 раз.

При воспроизведении изображения и картин на компьютере применяется "пуантилистский" способ (подобно технике художников-пуантилистов), т.е. изображение 
при помощи набора точек. Ясно, что в этом случае размерность $D=0$, и можно использовать формулу (15). Интересно, что этой формуле особенно хорошо отвечает "Мона Лиза" Леонардо да Винчи.

Теперь перейдем к случаю $\lambda_{i}=i^{2}$. Он возникает в задаче о спектральном разложении в ряд Фурье. Поскольку в этой задаче, как мы увидим, аналог $N_{i} / N$ есть очень большая величина, то для решения задачи достаточно знать оценку из леммы 1. Более того, окончательные формулы могут быть доказаны непосредственно, без перехода от дискретного варианта распределения типа Бозе-Эйнштейна (см. [8]). Мы, однако, хотим подчеркнуть здесь связь этих формул с бозе-статистикой.

Далее рассмотрим временной ряд $b_{1}, b_{2}, \ldots, b_{n}, \sum_{i=1}^{n} b_{i}^{2} \gg 1$. Используя теорему Котельникова, получаем разложение в ряд Фурье

$$
f(t)=\sum_{i=1}^{n} a_{i} \cos \left(\frac{\pi i t}{n}\right)
$$

обращающееся в целых точках $t=i$ в заданные значения $b_{i}, i=1,2, \ldots, n$. Будем предполагать, что:

а) $\frac{1}{n} \sum_{i=1}^{n} a_{i}^{2}=\frac{1}{n} \sum_{i=1}^{n} b_{i}^{2}=A_{0}, A_{0} n \gg 1$;

б) энергия ограничена, а именно

$$
\int_{0}^{2 \pi}\left(\frac{d f(t)}{d t}\right)^{2} d t \leqslant E, \quad E \gg 1 .
$$

Отсюда следует

$$
\sum_{i=1}^{n} i^{2} a_{i}^{2} \leqslant \frac{E n^{2}}{\pi^{2}} .
$$

Спектральной плотностью интервала $l$ называется средний квадрат амплитуды [9], а именно

$$
A_{l}=\frac{1}{n-l} \sum_{i=l}^{n} a_{i}^{2} .
$$

Если $a_{i}^{2}$ - рациональные числа, то их можно привести к общему знаменателю $N$ так, что $a_{i}^{2}=N_{i} / N$, где $N_{i}$ и $N$ целые. Тогда получим

$$
\sum_{i=1}^{n} \frac{N_{i}}{N}=A_{0} n
$$

Далее положим $i^{2}=\lambda_{i}$, тогда условие (16) примет вид

$$
\sum_{n=1}^{n} \lambda_{i} \frac{N_{i}}{N} \leqslant \frac{E n^{2}}{\pi^{2}} .
$$

Теперь условия “а” и “б” принимают обычный вид для теоремы, подобной сформулированной выше. Мы будем предполагать, что наборы $\left\{N_{i} / N\right\}_{i=1}^{n}$ равновероятны при ограничениях (17) и (18). 
Отсюда, поскольку

$$
A_{l}=\frac{1}{n-l} \sum_{i=l}^{n} \frac{N_{i}}{N}
$$

то, если $D$ известно, аналогично предыдущему (ср. (14) и (15)) для большинства вариантов $\left\{N_{i} / N\right\}$ (в смысле $\left.(14)\right)$ получим

$$
A_{l} \cong \frac{1}{n-l} \sum_{i=l}^{n} \frac{i^{D-1}}{\pi^{2} i^{2} \beta-\sigma} \sim \frac{1}{n-l} \int_{l}^{n} \frac{x^{D-1}}{\pi x^{2} \beta-\sigma} d x
$$

при условиях

$$
\int_{1}^{n} \frac{x^{1+D} d x}{\pi^{2} x^{2} \beta-\sigma}=\frac{E n^{2}}{\pi^{2}}, \quad \int_{1}^{n} \frac{x^{D-1} d x}{\pi^{2} x^{2} \beta-\sigma}=A_{0} n .
$$

По непрерывности эта асимптотика переносится на иррациональность.

Для колмогоровской турбулентности $D=-1 / 3$, а условие $A_{0} n \gg 1$ означает сильную внешнюю подкачку.

Эти формулы применимы также для уточнения поведения фликкер-шумов, когда известен главный член порядка убывания спектральной плотности, поскольку асимптотическое поведение (20) при $n-l \rightarrow \infty$ имеет вид $l^{D-1}$.

Теперь приведем связь формулы (20) с показателем Гёльдера (фрактальностью).

Рассмотрим произвольный интервал $[0, T]$ (стандартных) действительных чисел, $T>0$, и пусть $M$ - бесконечно большое нестандартное натуральное число. Эти числа далее фиксированы. Обозначим $d t=T / M$. Основное множество $\mathcal{T}=\{m d t \mid$ $0 \leqslant m \leqslant M\}$ содержит все стандартные числа из интервала $[0, T]$, а также те нестандартные действительные числа, которые отличаются от них на бесконечно малые величины.

Пусть $f$ - произвольная функция на $\mathcal{T}$ и $t \in \mathcal{T}-\{T\}$. Положим $d f(t)=f(t+d t)-$ $f(t)$.

ЛЕмма 2 [10]. Пусть $f$-непрерывная (возможно, принимающая нестандартнье значения) функция на $\mathcal{T}, p \in[1, \infty)$ - действительное число и пусть

$$
\operatorname{var}_{f}(p)=\sum_{t \in \mathcal{T}-\{T\}}|d f(t)|^{p}
$$

Тогда существует единственное действительное число $1 / H(f) \in[0,1]$ такое, что

1) $\operatorname{var}_{f}(p)$ - бесконечно большое число при $1 \leqslant p<1 / H(f)$;

2) $\operatorname{var}_{f}(p)$ - бесконечно малое число при $p>1 / H(f)$.

Число $H(f)$ будем называть показателем Гёльдера, а $1 / H(f)$ - вариационной размерностью. Если $H(f)=1 / 2$, то функция $f$ является винеровской. Мы воспользуемся соотношением между гладкостью функций и поведением на бесконечности 
ее преобразования Фурье, которое приведено в [5]. Известна связь между показателем Гёльдера $H$ стационарного случайного процесса и показателем $\alpha$ степенного убывания его спектра.

Пусть сигнал $f(t)$ стационарен, так что $\langle f(t)\rangle \equiv\langle f\rangle=$ const, и имеет показатель Гёльдера $H$, так что

$$
|f(t+s)-f(t)|^{2} \sim s^{2 H}, \quad 0<H<1 .
$$

Тогда для автокорреляционной функции

$$
F(s)=\langle f(t+s) f(t)\rangle-\left\langle f^{2}\right\rangle
$$

получаем

$$
F(0)-F(s) \sim s^{2 H}
$$

По теореме Винера-Хинчина спектральная плотность $J(\omega)$ является преобразованием Фурье автокорреляционной функции:

$$
J(\omega)=\int F(s) e^{i \omega s} d s=\int F(s) \cos \omega s d s
$$

и, соответственно,

$$
F(s)=\frac{1}{2 \pi} \int J(\omega) e^{-i \omega s} d \omega=\frac{1}{2 \pi} \int J(\omega) \cos \omega s d \omega
$$

Поскольку функция $F(s)$ четна, имеем

$$
F(0)-F(s)=\frac{1}{2 \pi} \int J(\omega)(1-\cos \omega s) d \omega=\frac{1}{\pi} \int J(\omega) \sin ^{2} \frac{\omega s}{2} d \omega .
$$

Пусть $J(\omega)$ убывает на бесконечности как $|\omega|^{-\alpha}$. Тогда

$$
F(0)-F(s) \sim \int|\omega|^{-\alpha} \sin ^{2} \frac{\omega s}{2} d \omega=s^{\alpha-1} \int|\omega s|^{-\alpha} \sin ^{2} \frac{\omega s}{2} d(\omega s) .
$$

Заменяя в последнем интеграле переменную на $u=\omega s$, видим, что зависимость от $s$ остается только в множителе перед интегралом. Таким образом, $s^{2 H} \sim F(0)-F(s) \sim$ $s^{\alpha-1}$ или

$$
H=\frac{1}{2}(\alpha-1), \quad \alpha=2 H+1 .
$$

Для колмогоровской турбулентности $H=1 / 3, D=-1 / 3$.

Во многих задачах вычисляется показатель Гёльдера для временных рядов. Здесь через спектральное разложение временного ряда дается уточнение показателя Гёльдера с помощью введения еще двух констант (двух показателей), которые дают хорошее приближение для не очень больших частот. 


\section{Список литературы}

[1] V.P. Maslov, Russ. J. Math. Phys., 14:1 (2007), 66-95.

[2] Yu. I. Manin, Bull. Amer. Math. Soc. (N.S.), 43:2 (2006), 139-161; math.AG/0502016.

[3] О. И. Завьялов, Перенормированные диаграммы Фейнмана, Наука, М., 1979.

[4] Л. Д. Ландау, Е. М. Лифшиц, Квантовая механика. Ч. І. Нерелятивистская теория, ОГИЗ, М.-Л., 1948.

[5] В. П. Маслов, Матем. заметки, 81:1 (2007), 157-160.

[6] В. П. Маслов, ТМФ, 150:3 (2007), 511-512.

[7] V.P. Maslov, Russ. J. Math. Phys., 13:3 (2006), 315-325.

[8] В. П. Маслов, В. Е. Назайкинский, Матем. заметки, 81:6 (2007), 879-892.

[9] У. Фриш, Турбулентность. Наследие А.Н. Колмогорова, ФАЗИС, М., 1998.

[10] G. Shafer, V. Vovk, Probability and Finance. It's Only a Game! Wiley Ser. Probab. Stat. Financial Eng. Sect., Wiley, New York, 2001. 